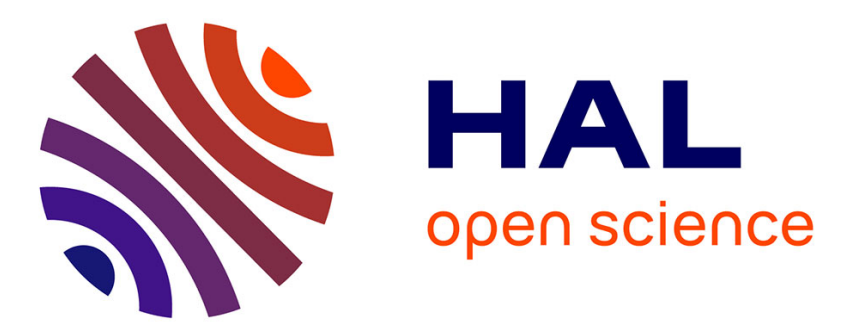

\title{
Efficient Data Structure for Representing and Simplifying Simplicial Complexes in High Dimensions
}

Dominique Attali, André Lieutier, David Salinas

\section{To cite this version:}

Dominique Attali, André Lieutier, David Salinas. Efficient Data Structure for Representing and Simplifying Simplicial Complexes in High Dimensions. SoCG 2011 - 27th Annual Symposium on Computational Geometry, Jun 2011, Paris, France. pp.s/n. hal-00579902

\section{HAL Id: hal-00579902 https://hal.science/hal-00579902}

Submitted on 25 Mar 2011

HAL is a multi-disciplinary open access archive for the deposit and dissemination of scientific research documents, whether they are published or not. The documents may come from teaching and research institutions in France or abroad, or from public or private research centers.
L'archive ouverte pluridisciplinaire HAL, est destinée au dépôt et à la diffusion de documents scientifiques de niveau recherche, publiés ou non, émanant des établissements d'enseignement et de recherche français ou étrangers, des laboratoires publics ou privés. 


\section{Efficient Data Structure for Representing and Simplifying Simplicial Complexes in High Dimensions}

\author{
Dominique Attali \\ Gipsa-lab \\ Grenoble, France \\ dominique.attali@gipsa- \\ lab.grenoble-inp.fr
}

\author{
[Extended Abstract]
}

\author{
André Lieutier \\ Dassault Système \\ Aix-en-Provence, France \\ andre.lieutier@3ds.com
}

\author{
David Salinas \\ Gipsa-lab \\ Grenoble, France \\ david.salinas@gipsa- \\ lab.grenoble-inp.fr
}

\begin{abstract}
We study the simplification of simplicial complexes by repeated edge contractions. First, we extend to arbitrary simplicial complexes the statement that edges satisfying the link condition can be contracted while preserving the homotopy type. Our primary interest is to simplify flag complexes such as Rips complexes for which it was proved recently that they can provide topologically correct reconstructions of shapes. Flag complexes (sometimes called clique complexes) enjoy the nice property of being completely determined by the graph of their edges. But, as we simplify a flag complex by repeated edge contractions, the property that it is a flag complex is likely to be lost. Our second contribution is to propose a new representation for simplicial complexes particularly well adapted for complexes close to flag complexes. The idea is to encode a simplicial complex $K$ by the graph $G$ of its edges together with the inclusion-minimal simplices in the set difference $\operatorname{Flag}(G) \backslash K$. We call these minimal simplices blockers. We prove that the link condition translates nicely in terms of blockers and give formulae for updating our data structure after an edge contraction. Finally, we observe in some simple cases that few blockers appear during the simplification of Rips complexes, demonstrating the efficiency of our representation in this context.
\end{abstract}

Categories and Subject Descriptors: F.2.2 [Analysis of Algorithms and Problem Complexity]: Nonnumerical Algorithms and Problems-Geometrical problems and computations, Computations on discrete structures; I.3.5 [Computer Graphics]: Computational Geometry and Object Modeling; E.2 [Data Storage Representations]: Object representation

\section{General Terms: Theory, Algorithms}

Keywords: Simplicial complexes, data structure, flag complexes, clique complexes, Vietoris-Rips complexes, shape reconstruction, shape simplification, edge contraction, homotopy equivalence, high dimensions

\footnotetext{
*This work is partially supported by ANR Project GIGA ANR-09BLAN-0331-01.
}

Permission to make digital or hard copies of all or part of this work for personal or classroom use is granted without fee provided that copies are not made or distributed for profit or commercial advantage and that copies bear this notice and the full citation on the first page. To copy otherwise, to republish, to post on servers or to redistribute to lists, requires prior specific permission and/or a fee.

SCG'11, June 13-15, 2011, Paris, France.

Copyright 2011 ACM 978-1-4503-0682-9/11/06 ...\$10.00.

\section{INTRODUCTION}

As datasets are growing larger in size and in dimension, simplicial complexes built upon these data become gigantic, challenging our ability to extract useful and concise information. In particular, storing all simplices becomes prohibitive. A way to overcome this difficulty is reducing the size of the simplicial complex prior to analysis. During that process, it is desirable to preserve the homotopy type.

In this work, we focus on the simplification of a particular class of simplicial complexes, likely to be encountered in high dimensional data analysis and manifold learning. Specifically, we are interested in flag complexes also known as clique complexes that have the property of containing simplices wherever the adjacency of vertices permit one. Precisely, the flag complex of a graph $G$ is the largest simplicial complex whose 1-skeleton is $G$. Obviously, flag complexes are completely determined by their 1 -skeletons, which provide a very light form of storage. A standard way of building the 1-skeleton of a flag complex is to consider the proximity graph of a point cloud. The flag complex of such a graph is called a Rips complex. In the light of recent results [1, 2], Rips complexes seem to be good candidates for reproducing the homotopy type of the shape sampled by the point cloud. In this context, simplification can be used as a preprocessing phase for reducing, for instance, the cost of computing topological invariants such as Betti numbers [6] 12]).

Following what has been done within the computer graphics and visualization communities, one can consider several elementary operations for simplifying a simplicial complex: vertex removal [14], vertex clustering [13], triangle contraction [8]. We primarily concentrate here on edge contraction, the operation that consists in merging two vertices. It was used in the pioneering work of Hoppe et al. [9] for generating progressive meshes and has been intensively studied ever since. Garland and Heckbert [7] proposed an elegant way of prioritizing edge contractions for surface simplification. Dey et al. [4] introduced a local condition called the link condition that characterizes edge contractions that permit a homeomorphic modification of 2- and 3-manifolds.

It would be tempting as we repeatedly apply edge contractions on a flag complex to keep its nature of flag complex, thus preserving its light form of storage along the simplification process. As already observed in [16] and confirmed by our first experiments, this seems to be "almost" possible. Indeed, Zomorodian uses a representation by simplicial sets that allows him to collapse any edge while keeping the homotopy type unchanged. He observed that, along the simplification process, most cells remain regular simplices. We 
suggest here another strategy that will preserve the representation by simplicial complexes along the simplification.

Our first contribution is the proof that the link condition introduced in [4] can also be used to guarantee homotopy-preserving edge contractions in arbitrary simplicial complexes. Our second contribution is to introduce a new data structure well-adapted for high-dimensional simplicial complexes which are "almost" flag complexes. Besides the 1-skeleton, we encode parsimoniously how the complex differs from the flag complex of its 1-skeleton. Precisely, we represent any simplicial complex $K$ by its 1 -skeleton $G$ together with the set of inclusion-minimal simplices in the set difference $\operatorname{Flag}(G) \backslash K$. These minimal simplices are called blockers. The intuition is that simplicial complexes "close" to flag complexes will have a small amount of blockers.

We show that the link condition translates nicely in terms of blockers in our new data structure and give an explicit expression of the blockers created (and destroyed) during an edge contraction. We have implemented the data structure and the edge contraction operation. Our first experiments indicate that the simplification of Rips complexes in some simple cases and using a reasonable strategy for prioritizing edge contractions leads to the apparition of very few blockers. This seems to make the proposed representation efficient in practice.

When drawing simplicial complexes in figures, we adopt the convention that besides drawing 1-skeletons, either we shade inclusionmaximal simplices or hatch blockers. When no triangles are shaded or hatched, the convention is that the blocker set is empty or equivalently that the simplicial complex is a flag complex.

\section{BASIC DEFINITIONS}

In this section, we recall standard definitions and notations that can be found in textbooks such as [11]. The cardinality of a set $X$ will be denoted $\sharp X$.

\subsection{Abstract simplicial complexes}

An abstract simplex is any finite non-empty set. The dimension of a simplex $\sigma$ is one less than its cardinality, $\operatorname{dim} \sigma=\sharp \sigma-1$. A $k$-simplex designates a simplex of dimension $k$. If $\tau \subset \sigma$ is a non-empty subset, we call $\tau$ a face of $\sigma$ and $\sigma$ a coface of $\tau$. If in addition $\tau \subsetneq \sigma$, we say that $\tau$ is a proper face and $\sigma$ is a proper coface. An abstract simplicial complex is a collection of simplices, $K$, that contains, with every simplex, the faces of that simplex. The dimension of $K$ is the largest dimension of one of its simplices. The closure of a set of simplices $\Sigma$, denoted $\mathrm{Cl}(\Sigma)$, is the smallest simplicial complex containing $\Sigma$. The vertex set of the abstract simplicial complex $K$ is the union of its elements, $\operatorname{Vert}(K)=\bigcup_{\sigma \in K} \sigma$. A subcomplex of $K$ is a simplicial complex $L \subset K$. A particular subcomplex is the $i$-skeleton consisting of all simplices of dimension $i$ or less, which we denote by $K^{(i)}$. The 0 skeleton is the set of inclusion-minimal simplices. Besides classical definitions, the following concept will be useful:

DEFINITION 1 (EXPANSION). Let $K$ be a simplicial complex whose dimension is $k$. The expansion of $K$, denoted Expand $(K)$, is the largest simplicial complex having $K$ as a $k$-skeleton. In particular, the expansion of a graph $G$ is the flag complex of $G$ and is denoted $\operatorname{Flag}(G)$.

Throughout the paper, we will restrict ourselves to finite simplicial complexes. Note that the expansion of the 0-skeleton $K^{(0)}$ is the power set of the vertex set $\operatorname{Vert}(K)$ minus the empty set, Expand $\left(K^{(0)}\right)=2^{\operatorname{Vert}(K)} \backslash\{\emptyset\}$ and consists of all simplices $\sigma \subset \operatorname{Vert}(K)$ spanned by vertices in $K$. In particular, it has a unique inclusion-maximal simplex which is $\operatorname{Vert}(K)$. In Section 4 we will be interested by expansions of $i$-skeletons, Expand $\left(K^{(i)}\right)$, which form for increasing values of $i$ an inclusion-decreasing sequence of simplicial complexes all containing $K$; see Figure 4 for a schematic drawing of $K$ and the expansion of its 0 - and 1skeletons.

\subsection{Intersection and union}

Two abstract simplices $\tau$ and $\sigma$ are disjoint if they have no vertices in common or equivalently if $\tau \cap \sigma=\emptyset$. It will be convenient to denote the union of two simplices $\sigma$ and $\tau$ simply $\sigma \tau$ instead of $\sigma \cup \tau$. In the same spirit, we shall use indifferently one of the two notations $\left\{v_{0}, v_{1}, \ldots, v_{k}\right\}$ or $v_{0} v_{1} \ldots v_{k}$ to designate the $k$ simplex spanned by vertices $v_{0}, v_{1}, \ldots, v_{k}$. In particular, we shall make no distinction between the vertex $v$ of $K$ and the 0 -simplex $\{v\} \in K$. We shall also use the notation $a b$ instead of $\{a, b\}$ to designate the edge connecting vertex $a$ and vertex $b$.

\subsection{Underlying space}

Let $\pi: \operatorname{Vert}(K) \rightarrow \mathbb{R}^{n}$ be an injective map that sends the $n$ vertices of $K$ to $n$ affinely independent points of $\mathbb{R}^{n}$, such as for instance the $n$ vectors of the standard basis of $\mathbb{R}^{n}$. The underlying space of $K$ is the point set $|K|=\bigcup_{\sigma \in K} \operatorname{Hull} \pi(\sigma)$ and is defined up to a homeomorphism. We shall say that a transformation $f$ between two simplicial complexes $K$ and $K^{\prime}$ preserves the topological type if the underlying spaces of $K$ and $K^{\prime}$ are homeomorphic, $|K| \approx\left|K^{\prime}\right|$ and we say that $f$ preserves the homotopy type if the underlying spaces are homotopy equivalent, $|K| \simeq\left|K^{\prime}\right|$.

\section{HOMOTOPY-PRESERVING EDGE CON- TRACTION}

In this section, we give a local condition on the link of an edge $a b$ in a simplicial complex $K$ under which the contraction of the edge $a b$ preserves the homotopy type of $K$. This condition, called the link condition, was introduced in [4] to characterize edge contractions that permit a homeomorphic modification when the simplicial complex $K$ is the triangulation of a 2-manifold or a 3-manifold. Unlike previous works [4. 15], we make no assumptions on the simplicial complex $K$. In particular, we do not require that $K$ triangulates a manifold.

\subsection{Edge contraction}

Recall that $\operatorname{Vert}(K)$ designates the set of vertices of $K$ and consider $a, b \in \operatorname{Vert}(K)$ and $c \notin \operatorname{Vert}(K)$. To describe the edge contraction $a b \mapsto c$, we define a vertex map $f$ that takes vertices $a$ and $b$ to $c$ and takes all other vertices to themselves:

$$
f(v)= \begin{cases}c & \text { if } v \in\{a, b\}, \\ v & \text { if } v \notin\{a, b\} .\end{cases}
$$

We then extend $f$ to all simplices $\sigma=\left\{v_{0}, \ldots, v_{k}\right\}$ of $K$, setting $f(\sigma)=\left\{f\left(v_{0}\right), \ldots, f\left(v_{k}\right)\right\}$. The edge contraction $a b \mapsto c$ is the operation that changes $K$ to $K^{\prime}=\{f(\sigma) \mid \sigma \in K\}$. By construction $f$ is surjective and $K^{\prime}$ is a simplicial complex. Note that the edge contraction $a b \mapsto c$ is well defined even when $a b$ does not belong to $K$.

\subsection{Link condition}

Let $\sigma$ be a simplex of the simplicial complex $K$. The link of $\sigma$ in $K$ is the simplicial complex

$$
\operatorname{Lk}_{K}(\sigma)=\{\tau \in K \mid \tau \cup \sigma \in K, \tau \cap \sigma=\emptyset\} .
$$


When $K$ is clear from the context, we will drop it and denote the link of $\sigma$ in $K$ simply by $\operatorname{Lk}(\sigma)$. In particular, whenever we contract an edge $a b \in K$ to a new vertex $c \in K^{\prime}$, it is unambiguous to write $\operatorname{Lk}(a), \operatorname{Lk}(b), \operatorname{Lk}(a b)$ for the links of $a, b$ and $a b$ in $K$ and $\operatorname{Lk}(c)$ for the link of $c$ in $K^{\prime}$. Before stating our main theorem, we start with two technical lemmas about links. We also review the Nerve Theorem on which relies our proof.

LEMmA 1. Let $\sigma \subset \operatorname{Vert}(K) \backslash\{a, b\}$ be a simplex spanned by vertices of $K$ disjoint from $a$ and $b$. The simplex co belongs to $K^{\prime}$ if and only if either a $\sigma$ or b $\sigma$ belongs to K. Equivalently, $\sigma \in \operatorname{Lk}(c)$ if and only if $\sigma \in \operatorname{Lk}(a) \cup \operatorname{Lk}(b)$.

Proof. Using $f^{-1}[\{c \sigma\}]=\{a \sigma, b \sigma, a b \sigma\} \cap K$, we get that $c \sigma \in K^{\prime} \Longleftrightarrow f^{-1}[\{c \sigma\}] \neq \emptyset \Longleftrightarrow\{a \sigma, b \sigma, a b \sigma\} \cap K \neq$ $\emptyset \Longleftrightarrow\{a \sigma, b \sigma\} \cap K \neq \emptyset \Longleftrightarrow a \sigma \in K$ or $b \sigma \in K$.

The star of a simplex $\sigma$ in $K$, denoted $\operatorname{St}_{K}(\sigma)$, is the collection of simplices of $K$ having $\sigma$ as a face. Provided that there is a unique inclusion-maximal simplex $\tau \neq \sigma$ in the star of $\sigma$, it is well-known that $|K|$ deformation retracts to $\left|K \backslash \mathrm{St}_{K}(\sigma)\right|$ and the operation that removes $\operatorname{St}_{K}(\sigma)$ is then called a collapse [5]. A simplicial complex is said to be collapsible if it can be reduced to a single vertex by a finite sequence of collapses. In particular, the underlying space of a collapsible complex is contractible.

Lemma 2. Suppose $a b \in K$ satisfies $\operatorname{Lk}(a b)=\operatorname{Lk}(a) \cap \operatorname{Lk}(b)$ and let $K^{\prime}$ be the simplicial complex obtained after the edge contraction $a b \mapsto c$. The preimage of the closure of any simplex in $K^{\prime}$ is non-empty and collapsible.

PROOF. For all $\sigma \in K^{\prime}$, we give an expression of the preimage $f^{-1}[\mathrm{Cl}(\sigma)]$ which entails its collapsibility. Recalling that the closure of a simplex is $\mathrm{Cl}(\sigma)=\bigcup_{\emptyset \neq \tau \subset \sigma}\{\tau\}$ and noting that the preimage of a union is the union of the preimages, we consider three cases:

Case 1: $f^{-1}[\mathrm{Cl}(c)]=f^{-1}[\{c\}]=\{a, b, a b\}$ is collapsible.

Case 2: If $\sigma \cap c=\emptyset$, then for all faces $\tau$ of $\sigma$, we also have $\overline{\tau \cap c}=\emptyset$ and therefore $f^{-1}[\{\tau\}]=\{\tau\}$. It follows that

$f^{-1}[\mathrm{Cl}(\sigma)]=\bigcup_{\emptyset \neq \tau \subset \sigma} f^{-1}[\{\tau\}]=\bigcup_{\emptyset \neq \tau \subset \sigma}\{\tau\}=\mathrm{Cl}(\sigma)$.

Case 3: If $\sigma$ belongs to the link of $c$ in $K^{\prime}$, then $\sigma \in \operatorname{Lk}(a) \cup$ $\operatorname{Lk}(b)$ by Lemma 1 If in addition $a b$ satisfies the link condition $\operatorname{Lk}(a b)=\operatorname{Lk}(a) \cap \operatorname{Lk}(b)$, this implies that $\sigma$ belongs either to $\operatorname{Lk}(a b)$ or to $\operatorname{Lk}(a) \backslash \operatorname{Lk}(a b)$ or to $\operatorname{Lk}(b) \backslash \operatorname{Lk}(a b)$. Observing that the same is true for all faces $\tau$ of $\sigma$, we deduce immediately that for all $\emptyset \neq \tau \subset \sigma$

$$
f^{-1}[\{c \tau\}]= \begin{cases}\{a \tau, b \tau, a b \tau\} & \text { if } \tau \in \operatorname{Lk}(a b), \\ \{a \tau\} & \text { if } \tau \in \operatorname{Lk}(a) \backslash \operatorname{Lk}(a b), \\ \{b \tau\} & \text { if } \tau \in \operatorname{Lk}(b) \backslash \operatorname{Lk}(a b) .\end{cases}
$$

Since $\mathrm{Cl}(c \sigma)=\{c\} \cup \mathrm{Cl}(\sigma) \cup \bigcup_{\emptyset \neq \tau \subset \sigma}\{c \tau\}$, we obtain that

$$
f^{-1}[\mathrm{Cl}(c \sigma)]=\{a, b, a b\} \cup \mathrm{Cl}(\sigma) \cup \underset{\emptyset \neq \tau \subset \sigma}{\bigcup} f^{-1}[\{c \tau\}] .
$$

Writing $\Sigma \cdot \Sigma^{\prime}=\left\{\sigma \sigma^{\prime} \mid \sigma \in \Sigma, \sigma^{\prime} \in \Sigma\right\}$ and setting $L=$ $\mathrm{Cl}(\sigma) \cap \mathrm{Lk}(a b)$ we get that $f^{-1}[\mathrm{Cl}(c \sigma)]$ is equal to

$$
\begin{cases}\mathrm{Cl}(a b \sigma) & \text { if } \sigma \in \operatorname{Lk}(a b), \\ \mathrm{Cl}(a \sigma) \cup\{b, a b\} \cup\{b, a b\} \cdot L & \text { if } \sigma \in \operatorname{Lk}(a) \backslash \operatorname{Lk}(a b), \\ \mathrm{Cl}(b \sigma) \cup\{a, a b\} \cup\{a, a b\} \cdot L & \text { if } \sigma \in \operatorname{Lk}(b) \backslash \operatorname{Lk}(a b) .\end{cases}
$$

Hence, if $\sigma \in \mathrm{Lk}(a b)$, the preimage $f^{-1}[\mathrm{Cl}(c \sigma)]$ is clearly collapsible. If $\sigma \in \operatorname{Lk}(a) \backslash \operatorname{Lk}(a b)$, we can always find a set of simplices $\lambda_{1}, \ldots, \lambda_{k}$ whose closure is equal to the simplicial complex $L=\mathrm{Cl}(\sigma) \cap \operatorname{Lk}(a b)$ and such that, for all $1 \leq i, j \leq k$, the simplex $\lambda_{i}$ is neither a face nor a coface of the simplex $\lambda_{j}$. In other words, the set of simplices $\lambda_{1}, \ldots, \lambda_{k}$ are the inclusion-maximal simplices of $L$. By construction, $a b \lambda_{i}$ is the only proper coface of $b \lambda_{i}$ in $f^{-1}[\mathrm{Cl}(c \sigma)]$. After a sequence of $k$ elementary collapses consisting in removing pairs of simplices $\left(b \lambda_{i}, a b \lambda_{i}\right)$, we are left with the simplicial complex $\mathrm{Cl}(a \sigma) \cup\{b, a b\}$ which is collapsible. The case $\sigma \in \operatorname{Lk}(b) \backslash \mathrm{Lk}(a b)$ is done similarly.

Recall that the nerve of a collection of set $\left\{S_{i}, i \in I\right\}$ is the simplicial complex

$$
\operatorname{Nrv}\left\{S_{i}, i \in I\right\} \quad=\quad\left\{\sigma \subset I \mid \bigcap_{i \in \sigma} S_{i} \neq \emptyset\right\}
$$

The Nerve Theorem has several versions [3], one of the earliest being due to Leray [10]. For the proof of Theorem 2 we shall use the following form:

THEOREM 1 (NERVE THEOREM). If a triangulable space $X$ is the union of a finite collection of closed sets $\left\{S_{i}, i \in I\right\}$, and if, for every $\sigma \in \operatorname{Nrv}\left\{S_{i}, i \in I\right\}, \bigcap_{i \in \sigma} S_{i}$ is contractible, then the underlying space of $\operatorname{Nrv}\left\{S_{i}, i \in I\right\}$ is homotopy equivalent to $X$.

We are now ready to state our main result (see Figure 1):
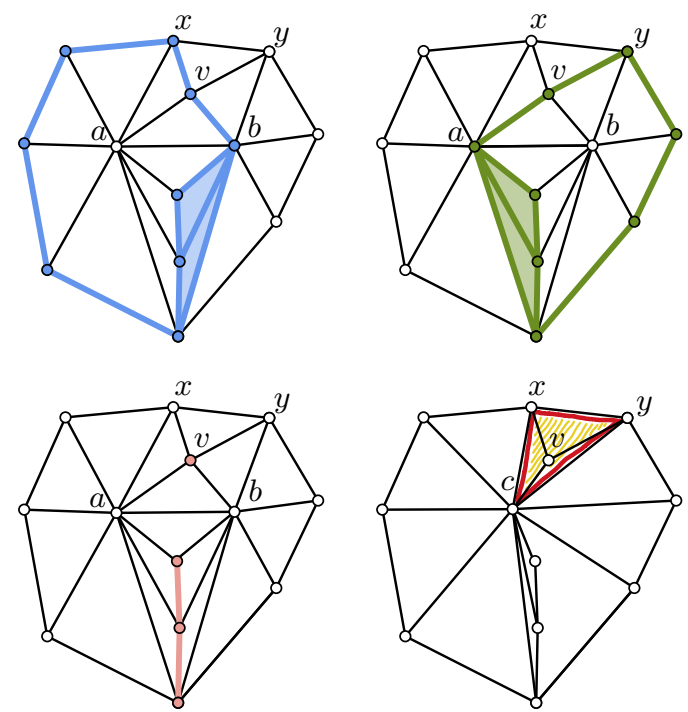

Figure 1: Top: link of $a$ and link of $b$. Bottom: link of $a b$ and simplicial complex after contraction of the edge $a b$. One can check that $a b$ satisfies the link condition. Equivalently, no blocker contains $a b$ (see Section 4.3. As a consequence, the contraction of $a b$ preserves the homotopy type. Note that the edge contraction $a b \mapsto c$ creates the blocker $c x y$ and $\alpha=x$ and $\beta=y$ satisfy $(i)$ and (ii) in Lemma 4. The contraction of any of the edges $c v, x v$ and $y v$ removes blocker $c x y$.

THEOREM 2 (LINK CONDITION THEOREM). Let $K$ be a simplicial complex. The contraction of the edge $a b \in K$ preserves the homotopy type whenever $\operatorname{Lk}(a b)=\operatorname{Lk}(a) \cap \operatorname{Lk}(b)$.

Proof. Suppose $a b \in K$ satisfies the link condition $\operatorname{Lk}(a b)=$ $\operatorname{Lk}(a) \cap \operatorname{Lk}(b)$ and let $K^{\prime}$ be the complex obtained after the edge 
contraction $a b \mapsto c$. The proof considers two coverings, one for $|K|$ and one for $\left|K^{\prime}\right|$, whose nerves $N$ and $N^{\prime}$ are proved to be equal and for which we establish that $|K| \simeq|N|$ and $\left|N^{\prime}\right| \simeq\left|K^{\prime}\right|$. By abuse of language, we will write $|\sigma|$ for the underlying space of the closure of $\sigma$.

Consider first the collection of sets $\left\{|\sigma|, \sigma \in K^{\prime}\right\}$ which covers $\left|K^{\prime}\right|$ and let $N^{\prime}$ denote its nerve. Clearly, for any simplex $\Sigma \in$ $N^{\prime}$, the intersection $\bigcap_{\sigma \in \Sigma} \sigma$ is either empty or a simplex of $K^{\prime}$ and therefore the intersection of sets in the collection $\bigcap_{\sigma \in \Sigma}|\sigma|$ is either empty or contractible. The Nerve Theorem then implies that $\left|K^{\prime}\right| \simeq\left|N^{\prime}\right|$. Let $\bar{f}:|K| \rightarrow\left|K^{\prime}\right|$ be the simplicial map induced by the vertex map $f: \operatorname{Vert}(K) \rightarrow \operatorname{Vert}\left(K^{\prime}\right)$ defined in Equation (1). Consider the collection of sets $\left\{\bar{f}^{-1}[|\sigma|], \sigma \in K^{\prime}\right\}$ obtained by taking the preimages of sets in the first collection. This collection covers $|K|$ and we denote its nerve by $N$. The two nerves $N$ and $N^{\prime}$ are equal because the surjectivity of $\bar{f}$ implies that:

$$
\bigcap_{\sigma \in \Sigma} \bar{f}^{-1}[|\sigma|]=\bar{f}^{-1}\left[\bigcap_{\sigma \in \Sigma}|\sigma|\right] \neq \emptyset \Longleftrightarrow \bigcap_{\sigma \in \Sigma}|\sigma| \neq \emptyset .
$$

Furthermore, if the intersection $\bigcap_{\sigma \in \Sigma}|\sigma|$ is non-empty, then there exists a simplex $\tau \in K^{\prime}$ such that $\tau=\bigcap_{\sigma \in \Sigma} \sigma$ and by Lemma 2 the intersection $\bigcap_{\sigma \in \Sigma} \bar{f}^{-1}[|\sigma|]=\bar{f}^{-1}[|\tau|]=\left|f^{-1}(\mathrm{Cl}(\tau))\right|$ is contractible since $f^{-1}(\mathrm{Cl}(\tau))$ is collapsible. To summarize, we established that $|K| \simeq|N| \approx\left|N^{\prime}\right| \simeq\left|K^{\prime}\right|$, showing that $|K|$ and $\left|K^{\prime}\right|$ have same homotopy type.

An example of edge $a b$ satisfying the link condition $\operatorname{Lk}(a b)=$ $\operatorname{Lk}(a) \cap \operatorname{Lk}(b)$ is given in Figure 1 where simplicial complexes are depicted with the convention adopted at the end of the introduction. Note that the converse of Theorem 2 is in general not true (see Figure 2,.
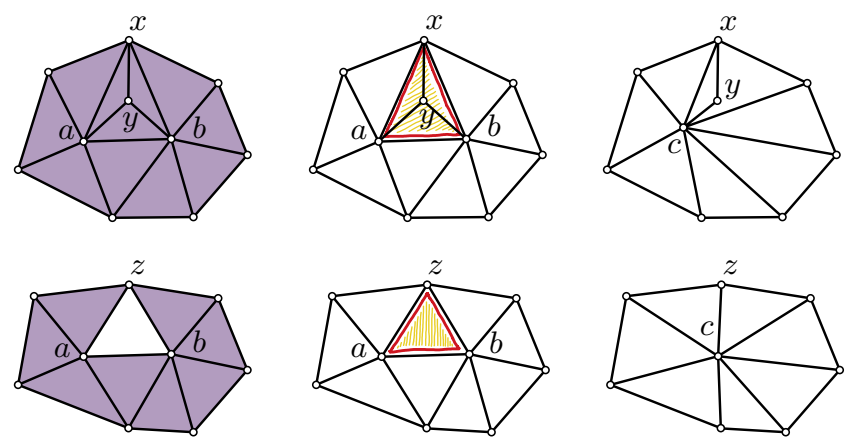

Figure 2: Left and middle: two 2-dimensional simplicial complexes with a blocker through $a b$. Right: The edge contraction $a b \mapsto c$ may (top) or may not (bottom) preserves the homotopy type.

\section{ENCODING COMPLEXES WITH THEIR SKELETONS AND BLOCKER SETS}

It is common to represent a simplicial complex $K$ of small dimension by the subset $L \subset K$ of simplices that are inclusionmaximal, that is, the set of simplices of $K$ which have no proper cofaces in $K$ (see Figure 3 top left). The simplicial complex $K$ can then be recovered from $L$ by taking the closure, $K=\mathrm{Cl}(L)$. In this section, we introduce a new way of representing simplicial complexes (see Figures 3 to 5 . Roughly, we store the 1-skeleton $G$ of $K$ together with a minimal set of simplices called blockers that indicates how much $K$ differs from the flag complex of $G$. First, we describe our data structure for encoding simplicial complexes. Then, we explain how to check the link condition and how to maintain the data structure as we contract edges. Pseudo-codes and time complexities are given in the appendix.

\subsection{Data structure}

DEFINITION 2 (BLOCKERS). Let $i \geq 0$. We say that a simplex $\sigma \subset \operatorname{Vert}(K)$ is an order $i$ blocker of $K$ if it satisfies the following three conditions (1) $\operatorname{dim} \sigma>i$; (2) $\sigma$ does not belong to $K$; (3) all proper faces of $\sigma$ belong to $K$. The set of order $i$ blockers of $K$ is denoted Blockers $_{i}(K)$.

Equivalently, the order $i$ blockers of $K$ are the inclusion-minimal simplices of Expand $\left(K^{(i)}\right) \backslash K$; see Figures 3 and 4
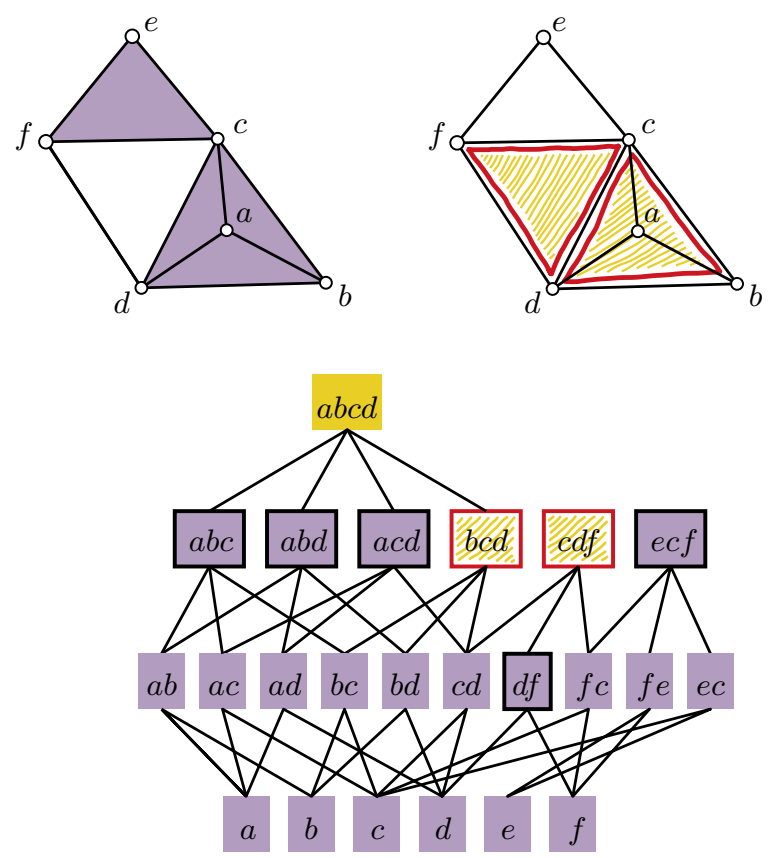

Figure 3: Top left: simplicial complex consisting of six vertices, ten edges and four non-overlapping shaded triangles. Top right: same simplicial complex represented by its 1-skeleton and order 1 blocker set $\{c d f, b c d\}$. Bottom: Hasse diagram of the expansion of the 1-skeleton. Nodes in dark gray are in the simplicial complex. Blockers and inclusion-maximal simplices are shown as framed nodes.

A key consequence is that the pair $\left(K^{(i)}, \operatorname{Blockers}_{i}(K)\right)$ encodes entirely the simplicial complex $K$. Indeed, the simplicial complex whose $i$-skeleton is $S$ and whose order $i$ blocker set is $B$ can be retrieved from the pair $(S, B)$ using the formula

$$
K=\{\sigma \in \operatorname{Expand}(S) \mid \sigma \text { has no face in } B\} .
$$

In this paper, we are primarily interested in simplicial complexes $K$ "close" to flag complexes with a "small" 1 -skeleton and therefore choose to represent them by the pair $\left(K^{(1)}\right.$, Blockers $\left.1(K)\right)$. Indeed, if $K=\operatorname{Flag}(G)$ is a flag complex, then its blocker set is empty and $K$ can be represented by the pair $(G, \emptyset)$. As we simplify the simplicial complex by edge contractions, we hope that the blocker set will remain small. This intuition is sustained by experiments we make in Section 5 in which $K$ is the Rips complex of a 


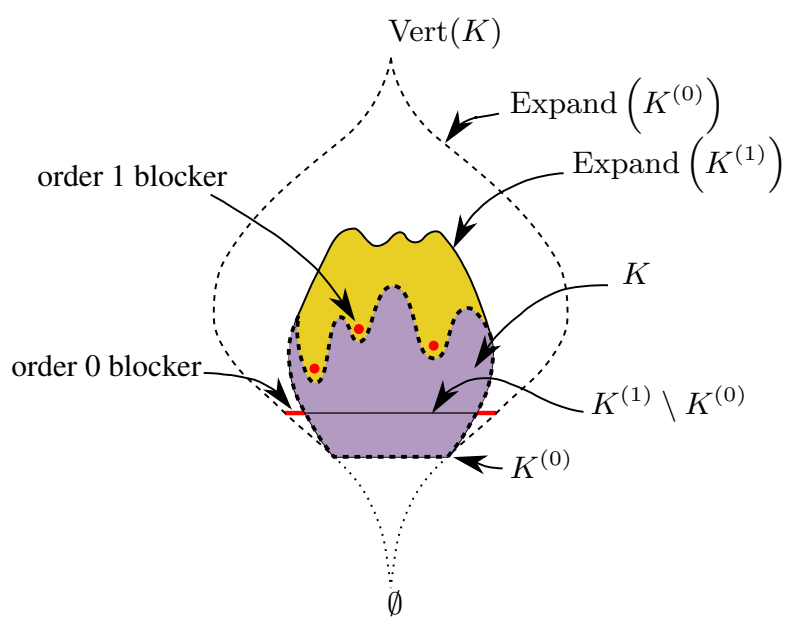

Figure 4: Hasse diagram of $K$.

point set that samples a shape. Hereafter, blockers will always refer to order 1 blockers.

Let $\mathcal{N}(v)=\mathcal{N}_{K}(v)$ be the set of vertices $w \neq v$ such that $v w \in K$ and write $\mathcal{B}(v)=\mathcal{B}_{K}(v)$ for the set of blockers that contain $v$. Clearly, encoding the pair $\left(K^{(1)}\right.$, $\left.\operatorname{Blockers}_{1}(K)\right)$ boils down to encoding for each vertex $v$ of $K$ the pair $(\mathcal{N}(v), \mathcal{B}(v))$. Precisely, our data structure consists of a linear array $V$ for the vertices and records for each vertex $v$ the set of neighbors $\mathcal{N}(v)$ and a set of pointers to blockers in $\mathcal{B}(v)$; see Figure 5 It follows that the size of our data structure is a constant times $\sum_{v \in \operatorname{Vert}(K)}(1+$ $\sharp \mathcal{N}(v)+2 \sharp \mathcal{B}(v))$. To see this, charge each vertex in a blocker to its corresponding vertex in $V$. During the operation, each vertex in $V$ is charged at most $\sharp \mathcal{B}(v)$ times.

To conclude this section, we give a crude upper bound on the dimension and number of blockers in a simplicial complex with $n$ vertices. Consider a blocker $\sigma$ passing through a vertex $v$. Since $\sigma \subset\{v\} \cup \mathcal{N}(v)$, we get $\sharp \sigma \leq 1+\sharp \mathcal{N}(v)$ and therefore $\operatorname{dim} \sigma \leq$

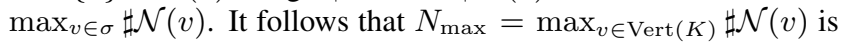
an upper bound on the dimension of the blockers and $O\left(2^{N_{\max }}\right)$ is an upper bound on the number of blockers through $v$. The total amount of blockers in our data structure is $O\left(n 2^{N_{\max }}\right)$.

\subsection{Testing whether a simplex belongs to the complex}

Recall that a simplex $\sigma$ belongs to $K$ if and only if $\sigma$ belongs to Expand $\left(K^{(i)}\right)$ and $\sigma$ has no face in $\operatorname{Blockers}_{i}(K)$ (see Equation (2)). It will be convenient to use this equivalence with $i=0$ for the proofs and $i=1$ for computations. Precisely, in the proofs, we will use that for all $\sigma \subset \operatorname{Vert}(K)$, we have the equivalence: $\sigma \in$ $K \Longleftrightarrow \sigma$ has no face in $\operatorname{Blockers}_{0}(K)$. For the computations, we will set $i=1$ and test whether $\sigma \subset \operatorname{Vert}(K)$ belongs to $K$ by checking whether its edges belong to the 1-skeleton and $\sigma$ contains no order 1 blocker; see the appendix for the details.

\subsection{Checking the Link Condition}

Next lemma formulates the link condition in terms of blockers.

Lemma 3. Let $K$ be a simplicial complex. The edge $a b \in K$ satisfies the link condition $\operatorname{Lk}(a b)=\operatorname{Lk}(a) \cap \operatorname{Lk}(b)$ if and only if no blocker of $K$ contains ab.

Proof. It is not difficult to see that for all simplicial complexes $K$ and for all edges $a b \in K$, we have the inclusion $\operatorname{Lk}(a b) \subset$

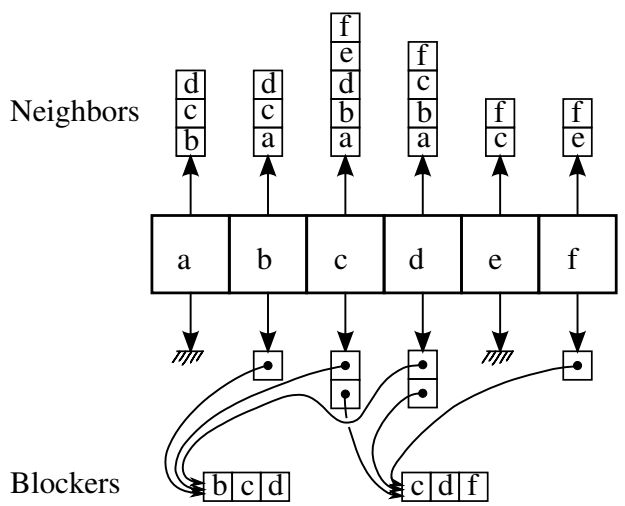

Figure 5: Data structure representing the simplicial complex in Figure 3 .

$\operatorname{Lk}(a) \cap \operatorname{Lk}(b)$. Let us prove that $\operatorname{Lk}(a b)=\operatorname{Lk}(a) \cap \operatorname{Lk}(b)$ implies that no blocker of $K$ contains $a b$. Suppose for a contradiction that the simplex $a b \tau$ is a blocker of $K$ for some simplex $\tau$ such that $a b \cap \tau=\emptyset$. By definition of a blocker, all proper faces of $a b \tau$ belong to $K$ and in particular $a \tau \in K$ and $b \tau \in K$. On the other hand, $a b \tau$ does not belong to $K$. It follows that $\tau \in \operatorname{Lk}(a), \tau \in$ $\operatorname{Lk}(b)$ and $\tau \notin \operatorname{Lk}(a b)$, implying that $\operatorname{Lk}(a b) \neq \operatorname{Lk}(a) \cap \operatorname{Lk}(b)$. Conversely, suppose no blocker of $K$ contains $a b$ and let us prove that $\operatorname{Lk}(a) \cap \operatorname{Lk}(b) \subset \operatorname{Lk}(a b)$. Consider a simplex $\sigma \in \operatorname{Lk}(a) \cap$ $\operatorname{Lk}(b)$. By definition, $a \sigma \in K, b \sigma \in K$ and $a b \cap \sigma=\emptyset$. We claim that all proper faces of $a b \sigma$ belong to $K$. If this claim is satisfied, then we are done. Indeed, since $a b \sigma$ cannot be a blocker, one has $a b \sigma \in K$ and therefore $\sigma \in \operatorname{Lk}(a b)$. We now prove the claim by contradiction. Suppose some of the proper faces of $a b \sigma$ do not belong to $K$ and let $\tau_{\min }$ be an inclusion-minimal face among them. In other words, $\tau_{\min }$ is an order 0 blocker. Since $\tau_{\min } \subset$ $a b \sigma, \tau_{\min } \notin K, a \sigma \in K$ and $b \sigma \in K$, we must have $a b \subset \tau_{\text {min }}$ which contradicts the assumption that no (order 1) blocker of $K$ contains $a b$.

Testing whether an edge $a b \in K$ satisfies the link condition can be done by traversing the blockers through $a$ and testing for each blocker whether it contains $b$. The pseudo-code and time complexity of this procedure are given in the appendix.

\subsection{Updating the data structure after an edge contraction}

In this section, we describe how to update the data structure after an edge contraction. More precisely, we consider a simplicial complex $K$ and let $K^{\prime}$ be the simplicial complex obtained after the edge contraction $a b \mapsto c$. Our goal is to compute the pair $\left(\mathcal{N}_{K^{\prime}}(c), \mathcal{B}_{K^{\prime}}(c)\right)$. Clearly, $\mathcal{N}_{K^{\prime}}(c)=(\mathcal{N}(a) \backslash\{b\}) \cup(\mathcal{N}(b) \backslash$ $\{a\})$. Next lemma prepares the computation of $\mathcal{B}_{K^{\prime}}(c)$ by characterizing blockers through $c$.

LEMMA 4. Let $K^{\prime}$ be the simplicial complex obtained after the edge contraction $a b \mapsto c$. Suppose $\sigma \subset \operatorname{Vert}(K) \backslash\{a, b\}$ is a simplex with $\operatorname{dim} \sigma \geq 1$. The simplex c $\sigma$ belongs to Blockers $1\left(K^{\prime}\right)$ if and only if the following two conditions are fulfilled:

(i) $\sigma \in K$; every proper face of $\sigma$ belongs to $\operatorname{Lk}(a) \cup \operatorname{Lk}(b)$;

(ii) $\sigma=\alpha \beta$ with $a \beta \in \operatorname{Blockers}_{0}(K)$ and $b \alpha \in \operatorname{Blockers}_{0}(K)$.

PROOF. First, note that the proper faces of $c \sigma$ belong to $K^{\prime}$ if and only if $(i)$ is satisfied. Indeed, using Lemma 1, $(i)$ is equivalent to $\sigma \in K^{\prime}$ and $c \tau \in K^{\prime}$ for all proper faces $\tau \subsetneq \sigma$. Hence, $c \sigma \in$ 
$\operatorname{Blockers}_{1}\left(K^{\prime}\right) \Longrightarrow(i)$. Let us prove that $c \sigma \in \operatorname{Blockers}_{1}\left(K^{\prime}\right)$ $\Longrightarrow$ (ii). Since $c \sigma \notin K^{\prime}$, neither $a \sigma$ nor $b \sigma$ belongs to $K$. It follows that $a \sigma$ has a face in $\operatorname{Blockers}_{0}(K)$ and since $\sigma \in K$, this face must contain $a$. Let us denote this face $a \beta$ with $\beta \subset$ $\sigma$. Similarly, since $b \sigma \notin K$, there exists a face $\alpha \subset \sigma$ such that $b \alpha \in \operatorname{Blockers}_{0}(K)$. Let us prove that $\alpha \beta=\sigma$. Suppose for a contradiction that $\alpha \beta$ is a proper face of $\sigma$. (i) implies that $\alpha \beta \in$ $\operatorname{Lk}(a) \cup \operatorname{Lk}(b)$. If $\alpha \beta$ belongs to $\operatorname{Lk}(a)$, then $\beta$ being a face of $\alpha \beta$ must also belong to $\operatorname{Lk}(a)$ which contradicts $a \beta \in \operatorname{Blockers}_{0}(K)$. Similarly, if $\alpha \beta$ belongs to $\operatorname{Lk}(b)$, we also get a contradiction.

Conversely, let us prove that $(i)$ and $(i i) \Longrightarrow c \sigma \in \operatorname{Blockers}\left(K^{\prime}\right)$. We have seen that $(i)$ implies that all proper faces of $c \sigma$ belongs to $K^{\prime}$. To prove that $c \sigma \notin K^{\prime}$, we note that neither $a \sigma$ nor $b \sigma$ belongs to $K$. Indeed, $a \sigma=a \alpha \beta \notin K$ since its face $a \beta \in \operatorname{Blockers}_{0}(K)$ and $b \sigma=b \alpha \beta \notin K$ since its face $b \alpha \in \operatorname{Blockers}_{0}(K)$.
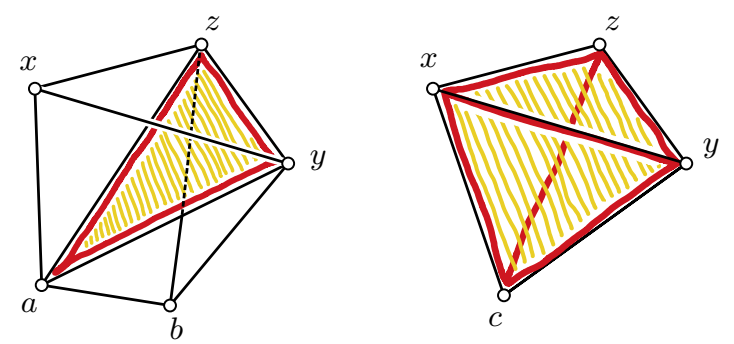

Figure 6: Triangle $a y z$ is a 2-blocker. We have that $\operatorname{Lk}(a)=$ $\{x, y, z, x y, x z, b, b y, b z\}, \operatorname{Lk}(b)=\{y, z, y z, a, a y, a z\}$, $\operatorname{Lk}(a b)=\{y, z\}$. Note that $\sigma=\alpha \beta$ with $\alpha=x$ and $\beta=y z$ fulfills $(i)$ and (ii) in Lemma 4 Therefore, the edge contraction $a b \mapsto c$ leads to the creation of the 3-blocker $c x y z$ and the destruction of the 2-blocker ayz.

A few remarks. Suppose $\sigma=\alpha \beta$ with $\operatorname{dim} \sigma \geq 1$ and $a b \cap$ $\sigma=\emptyset$ satisfies $(i)$ and $(i i)$ in Lemma 4 Because order 0 blockers have dimension 1 or more, the two sets $\alpha$ and $\beta$ are non-empty. Writing $d_{i}(v)$ for the largest dimension of order i blockers through $v$, it follows directly from the lemma that the largest dimension of order 1 blockers through $c$ satisfies $d_{1}(c) \leq d_{0}(a)+d_{0}(b)$ (see Figure 6 for an example in which equality is attained). Finally, we show that if $\alpha$ is a vertex, then $\alpha \in \mathcal{N}(a)$. Suppose $\alpha$ is a vertex. Because $\operatorname{dim} \sigma \geq 1, \alpha$ is a proper face of $\sigma$. Thus, (i) implies $\alpha \in \operatorname{Lk}(a) \cup \operatorname{Lk}(b)$ and (ii) implies $\alpha \notin \operatorname{Lk}(b)$, yielding $\alpha \in \operatorname{Lk}(a)$.

We are now ready to derive an expression for the set of blockers through $c$. First, note that

$$
\begin{aligned}
\operatorname{Blockers}_{0}(K) & =\operatorname{Blockers}_{1}(K) \\
& \cup\left\{x y \mid x \in K^{(0)}, y \in K^{(0)}, x y \notin K^{(1)}\right\} .
\end{aligned}
$$

Hence, $b \alpha \in \operatorname{Blockers}_{0}(K)$ if and only if $b \alpha \in \operatorname{Blockers}_{1}(K)$ or $\alpha \in K^{(0)} \backslash \mathcal{N}(b)$. Thus, to exhaust simplices $\sigma=\alpha \beta$ with $\operatorname{dim} \sigma \geq 1, a b \cap \sigma=\emptyset$ that satisfy (i) and (ii), it suffices to take $\alpha$ in the set of simplices

$$
\begin{array}{r}
Z_{a}(b)=\{\alpha \mid b \alpha \in \mathcal{B}(b), a b \cap \alpha=\emptyset\} \\
\cup(\mathcal{N}(a) \backslash(\mathcal{N}(b) \cup\{b\})) .
\end{array}
$$

Switching $a$ and $b$, we define $Z_{b}(a)$ similarly and obtain

$$
\begin{array}{r}
\mathcal{B}_{K^{\prime}}(c)=\left\{c \alpha \beta \mid \alpha \in Z_{a}(b), \beta \in Z_{b}(a), \alpha \beta \in K,\right. \\
\forall \tau \subsetneq \alpha \beta, \tau \in \operatorname{Lk}(a) \cup \operatorname{Lk}(b)\} .
\end{array}
$$

From this formula, we derive immediately an algorithm for computing $\mathcal{B}_{K^{\prime}}(c)$ presented in the appendix. The only piece that we still need to explain is how to compute the link of a vertex.

\subsection{Computing the link of a vertex}

Since the link of a vertex $v \in K$ is a simplicial complex, we can also represent it by a pair consisting of its 1-skeleton and its order 1 blocker set. We give below formulas expressing each element in the pair with respect to the 1-skeleton and order 1 blocker set of $K$.

Lemma 5. Let $K$ be a simplicial complex. For every vertex $v$ in $K$, we have:

$$
\begin{aligned}
& \operatorname{Lk}(v)^{(1)}=\left\{\sigma \subset \mathcal{N}(v) \mid \sigma \in K^{(1)}, v \sigma \notin \operatorname{Blockers}_{1}(K)\right\} \\
& \operatorname{Blockers}_{1}(\operatorname{Lk}(v))=\left\{\sigma \in K \mid v \sigma \in \operatorname{Blockers}_{2}(K)\right\} \\
& \cup\left\{\sigma \in \operatorname{Blockers}_{1}(K) \mid \forall \tau \subsetneq \sigma, v \tau \in K\right\}
\end{aligned}
$$

PROOF. Let us prove the first formula. By definition, $\sigma$ is a vertex or an edge of the link of $v$ if and only if $\sigma \in K^{(1)}, \sigma \cap v=\emptyset$ and $v \sigma \in K$. The latter condition is equivalent to $\sigma \subset \mathcal{N}(v)$, $\sigma \in K^{(1)}$ and $v \sigma \notin$ Blockers $_{1}(K)$, yielding the first formula.

Set $X=\left\{\sigma \in K \mid v \sigma \in\right.$ Blockers $\left._{2}(K)\right\}$ and $Y=\{\sigma \in$ Blockers $\left._{1}(K) \mid \forall \tau \subsetneq \sigma, v \tau \in K\right\}$. Note that no simplices $\sigma$ in $X \cup Y$ contain $v$. Indeed, suppose for a contradiction that $v \in \sigma$ and $\sigma \in X \cup Y$. Then, $v \sigma=\sigma$ and we would get simultaneously $\sigma \in K$ and $\sigma \notin K$, which is impossible. We establish the second formula by proving that Blockers $1(\operatorname{Lk}(v))=X \cup Y$. We first prove that Blockers $1(\operatorname{Lk}(v)) \subset X \cup Y$. Consider an order 1 blocker $\sigma$ of the link of $v$. By definition, $\operatorname{dim} \sigma \geq 2$

$$
\sigma \notin \operatorname{Lk}(v) \quad \text { and } \quad \forall \tau \subsetneq \sigma, \tau \in \operatorname{Lk}(v) .
$$

We distinguish two cases. If $\sigma \in K$, we deduce that every proper face of $v \sigma$ belongs to $K$ while on the other hand $v \sigma \notin K$. Thus, $v \sigma$ is a blocker of $K$ and $\sigma \in X$. If $\sigma \notin K$, observe that every proper face of $\sigma$ belongs to $K$. Thus, $\sigma$ is a blocker of $K$ and $\sigma \in Y$.

For the converse, recall that no simplices $\sigma \in X \cup Y$ contain $v$. Hence, for all faces $\tau$ of $\sigma$, we have $v \tau \in K \Longleftrightarrow \tau \in \operatorname{Lk}(v)$.

To prove that $X \subset$ Blockers $_{1}(\operatorname{Lk}(v))$, consider $\sigma \in X$. By definition, $\sigma \in K$ and $v \sigma$ is an order 2 blocker of $K$. This implies $v \sigma \notin K$ and for all $\tau \subsetneq \sigma, v \tau \in K$. Hence, $\sigma \notin \operatorname{Lk}(v)$ and for all $\tau \subsetneq \sigma, \tau \in \operatorname{Lk}(v)$, showing that $\sigma$ is an order 1 blocker of the link of $v$.

To prove that $Y \subset$ Blockers $_{1}(\operatorname{Lk}(v))$, consider $\sigma \in Y$. By definition, $\sigma$ is a blocker of $K$ whose proper faces belong to the link of $v$. Since $\sigma \notin K$, it follows that $\sigma \notin \operatorname{Lk}(v)$ and $\sigma$ is a blocker of $\operatorname{Lk}(v)$.

Note that for every vertex $x \in \operatorname{Lk}(v)$, we have $\sharp \mathcal{N}_{\mathrm{Lk}(v)}(x) \leq$ $\sharp \mathcal{N}_{K}(x)$ and $\sharp \mathcal{B}_{\operatorname{Lk}(v)}(x) \leq \sharp \mathcal{B}_{K}(x)$. In particular, if $K$ is a flag complex, so is the link of any of its vertices. The algorithm for computing the link together with its time complexity are given in the appendix.

Let $N_{\sigma}=\max _{v \in \sigma} \sharp \mathcal{N}(v)$. Overall, updating the data structure after the edge contraction $a b \mapsto c$ has a cost which increases with the number and dimension of blockers in a neighborhood of $a$ and $b$ and can be done efficiently in $O\left(N_{a} N_{b} \log N_{\mathcal{N}(a)}\right)$ assuming there are no blockers in a neighborhood of $a$ and $b$ (see the appendix for the details).

\section{EXPERIMENTS AND DISCUSSION}

In this section we apply our representation to the simplification of a subfamily of flag complexes, namely Rips complexes. Given as input a point cloud $P$ in a metric space and a real number $r \geq 0$, the 
proximity graph $G_{r}(P)$ is the graph whose vertices are the points $P$ and whose edges connect all pairs of points within distance $2 r$. By definition, the Rips complex is the flag complex of the proximity graph, $\mathcal{R}(P, r)=\operatorname{Flag}\left(G_{r}(P)\right)$. In our experiments, we consider finite point sets $P$ that sample various $d$-dimensional manifolds $X$ embedded in the $D$-dimensional Euclidean space. Typically, $d \in\{2,3\}$. Rips complexes are built using the extrinsic distance of the embedding space. When sampling shape $X$ with point set $P$, we try to adjust the Hausdorff distance $\varepsilon=d_{H}(P, X)$ and the scale parameter $r$, so that $|\mathcal{R}(P, r)| \simeq X$. In this we are helped by [2] which describes conditions guaranteeing that the Rips complex $\mathcal{R}(P, r)$ recovers the homotopy type of $X$, whenever $X$ has a positive $\mu$-reach for $\mu>0.775$ in $\mathbb{R}^{3}$ and $\mu>0.814$ in $\mathbb{R}^{4}$. Note that the number of neighbors of a vertex $v$ in the Rips complex are the number of points of $P$ in the ball centered at $v$ with radius $2 r$. If we assume no oversampling, an upper bound on the number of neighbors is $N_{\max }=O\left(\frac{r^{d}}{\varepsilon^{d}}\right)$. Since the number of blockers through $v$ is upper bounded by $2^{N_{\max }}$, we get that the size of our data structure depends only upon the intrinsic dimension $d$ and not upon the ambient dimension $D$.

We consider two scenarios for building $P$. First, we sample regularly the boundary of a cube, $C_{d}=\partial[-1,1]^{d+1}$. We then normalize these points to get a second point set that samples the $d$-sphere $S_{d}=\left\{x \in \mathbb{R}^{d+1} \mid\|x\|=1\right\}$. Once the Rips complex of $P$ is built, we simplify it by iteratively contracting edges. Initially, all edges are stored in a priority queue $\mathcal{Q}$. We use the length of the edges to prioritize them, so that the shortest edge has highest priority. We then remove the edge $a b$ with highest priority from the priority queue. If $a b$ satisfies the link condition, we contract $a b$ to a new vertex $c=\frac{a+b}{2}$ and update the data structure, which includes the removal of edges from $\mathcal{Q}$ and the insertion of new edges into $\mathcal{Q}$. We let the process continue until no edges remain in $\mathcal{Q}$. Each edge contraction decreases the number of vertices by one. We call $K_{i}$ the simplicial complex obtained after $i$ edge contractions and set $n_{i}=\sharp \operatorname{Vert}\left(K_{i}\right)=\sharp \operatorname{Vert}\left(K_{0}\right)-i$. For each point set $P$, we plot with respect to the number $i$ of edge contractions :

- the number of blockers in $K_{i}$ (first column in Figure 8).

- $\sum_{v \in K_{i}} \sharp \mathcal{N}(v), 2 \sum_{v \in K_{i}} \sharp \mathcal{B}(v)$ and the sum which is proportional to the size of the data structure (second column in Figure 8.

- $\frac{1}{n_{i}} \sum_{v \in K_{i}} \sharp \mathcal{N}(v)$ and $\frac{1}{n_{i}} \sum_{v \in K_{i}} \sharp \mathcal{B}(v)$ which represent respectively the average number of neighbors and the average number of blockers per vertex (third column in Figure 8).

- the average dimension of blockers in the complex (Figure 7).

In our experiments, we observe that the total amount of blockers increases at first but eventually diminishes and reaches 1 for our point sets that sample $C_{2}, S_{2}$ and $C_{3}$. The blocker after the last edge contraction is a tetrahedron for $C_{2}$ and $S_{2}$ and is a 4-simplex for $C_{3}$. Furthermore, this unique remaining blocker is spanned by the entire vertex set of the complex. The results we obtain are thus consistent with the topology of the sampled shape. We also observe that the size of our data structure decreases during the simplification, the additional cost of storing blockers remaining negligible compared to the cost of storing the neighbors. Furthermore, the average dimension of blockers remains close to 2 .

These very first illustrations of our data structure and simplification procedure are quite promising. Indeed, in these preliminary experiments we have only tested one of the simplest criteria for ordering edge contractions, namely the edge length, and restricted ourselves to a strict application of edge collapses. In fact, together with [2], we believe that this theoretical work lays theoretical foundations and opens a new field of design and experimentation of simplification strategies or computation of topological invariants in our representation. In our future work, usual simplification operations including, beyond edge contractions, cloud filtering or simplex collapses and anti-collapses will be revisited in this context.
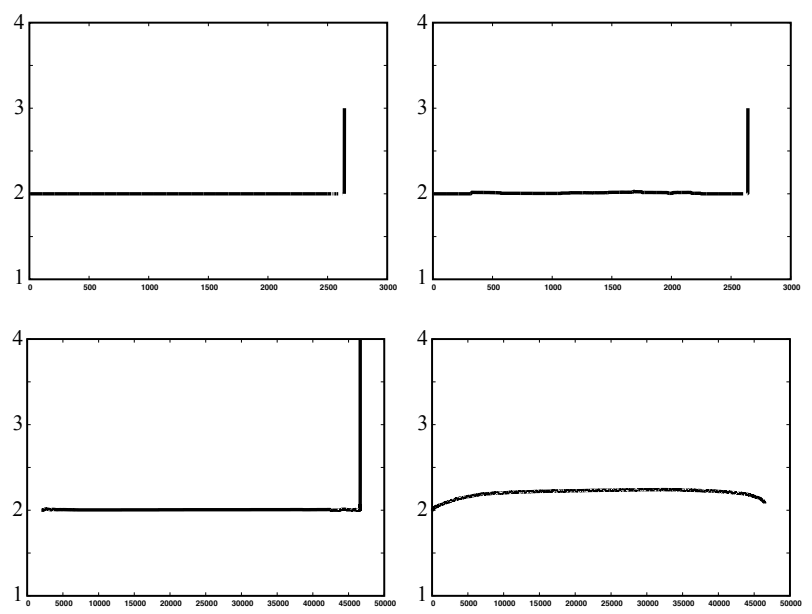

Figure 7: Average dimension of blockers for $C_{2}, S_{2}, C_{3}, S_{3}$.

\section{REFERENCES}

[1] D. Attali and A. Lieutier. Reconstructing shapes with guarantees by unions of convex sets. In 26th Ann. Sympos. Comput. Geom., pages 344-353, Snowbird, Utah, June 13-16 2010.

[2] D. Attali, A. Lieutier, and D. Salinas. Vietoris-Rips complexes also provide topologically correct reconstructions of sampled shapes. In Proc. 27th Ann. Sympos. Comput. Geom., Paris, France, June 13-15 2011.

[3] A. Bjorner. Topological methods. In Handbook of combinatorics (vol. 2), page 1850. MIT Press, 1996.

[4] T. Dey, H. Edelsbrunner, S. Guha, and D. Nekhayev. Topology preserving edge contraction. Publ. Inst. Math. (Beograd) (N.S.), 66:23-45, 1999.

[5] T. K. Dey, H. Edelsbrunner, and S. Guha. Computational topology. In B. Chazelle, J. E. Goodman, and R. Pollack, editors, Advances in Discrete and Computational Geometry, volume 223 of Contemporary Mathematics. AMS, Providence, 1999.

[6] H. Edelsbrunner and J. Harer. Computational topology: an introduction. Amer Mathematical Society, Providence, Rhode Island, 2010.

[7] M. Garland and P. Heckbert. Surface simplification using quadric error metrics. In Proceedings of the 24th annual conference on Computer graphics and interactive techniques, pages 209-216. ACM Press/Addison-Wesley Publishing Co., 1997.

[8] T. Gieng, B. Hamann, K. Joy, G. Schussman, and I. Trotts. Constructing hierarchies for triangle meshes. IEEE Transactions on Visualization and Computer Graphics, 4(2): 145-161, 2002.

[9] H. Hoppe. Progressive meshes. In Proceedings of the 23rd annual conference on Computer graphics and interactive techniques, pages 99-108. ACM, 1996.

[10] J. Leray. Sur la forme des espaces topologiques et sur les points fixes des représentations. J. Math. Pures Appl., 24:95-167, 1945.

[11] J. Munkres. Elements of algebraic topology. Perseus Books, 1993.

[12] P. Perry and V. de Silva. Plex: Simplicial complexes in MATLAB, 2006.

[13] J. Rossignac and P. Borrell. Multi-resolution 3D approximations for rendering complex scenes. In B. Falcidieno and T. Kunii, editors, Modeling in Computer Graphics: Methods and Applications, pages 455-465. Springer-Verlag, 1993.

[14] W. J. Schroeder, J. A. Zarge, and W. E. Lorensen. Decimation of triangle meshes. Computer Graphics (SIGGRAPH' 92 proceedings), 26(2):65-70, 1992.

[15] D. M. Thomas, V. Natarajan, and G.-P. Bonneau. Link conditions for simplifying meshes with embedded structures. IEEE Transactions on Visualization and Computer Graphics, 99, 2010.

[16] A. Zomorodian. The tidy set: a minimal simplicial set for computing homology of clique complexes. In Proceedings of ACM symposium of computational geometry, 2010. 

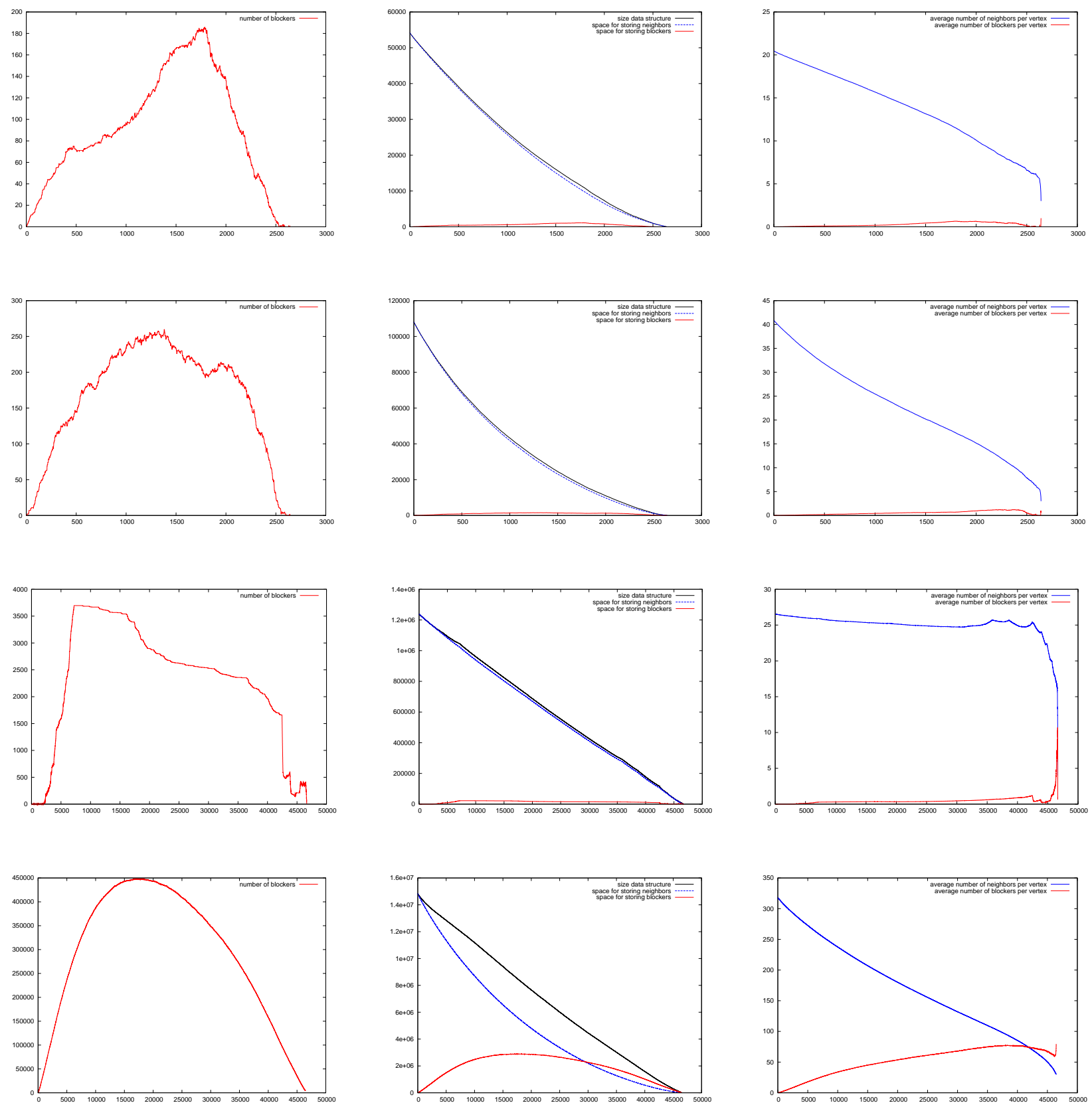

Figure 8: First row: point set $P$ of size 2646 that samples $C_{2}=\partial[-1,1]^{3}$ in $\mathbb{R}^{3}$ with $d_{H}\left(P, C_{2}\right) \leq 0.07$ and simplification of its Rips complex built at scale $r=0.2$. Second row: point set $P$ of size 2646 that samples the unit 2 -sphere $S_{2}$ in $\mathbb{R}^{3}$ with $d_{H}\left(P, S_{2}\right) \leq 0.07$ and simplification at scale $r=0.2$. Third row: point set $P$ of size 46656 that samples $C_{3}=\partial[-1,1]^{4}$ in $\mathbb{R}^{4}$ with $d_{H}\left(P, C_{3}\right) \leq 0.1$ and simplification at scale $r=0.2$. Fourth row: point set $P$ of size 46656 that samples $S_{3}$ in $\mathbb{R}^{4}$ with $d_{H}\left(P, S_{3}\right) \leq 0.1$ and simplification at scale $r=0.3$. At the end of the simplification, the simplicial complex possesses 1 blocker for the first three point sets and 184 vertices and 4758 blockers with average dimension 2.08 for the last point set. The number of blockers dramatically drops in the last experiment if we take $r=0.2$ 


\section{APPENDIX}

We provide pseudo-codes for testing the link condition, computing the link of a vertex $v$, testing whether a vertex belongs to a complex and computing the blockers passing through $c$ after the edge contraction $a b \mapsto c$. To express time complexities, let $N_{\sigma}=$ $\max _{v \in \sigma} \sharp \mathcal{N}(v)$ and $B_{\sigma}=\max _{v \in \sigma} \sharp \mathcal{B}(v)$. Moreover, let $M_{v}$ be the number of blockers with at least one vertex in $\mathcal{N}(v)$ and $M_{v}^{\prime} \leq M_{v}$ be the number of blockers contained in $\mathcal{N}(v)$. Recall that $d_{i}(v)$ designates the largest dimension of order $i$ blockers through $v$. We also suppose that we are able to answer whether $v \in \sigma$ in $O(\log \sharp \sigma)$ time and whether $\tau \subset \sigma$ in $O(\sharp \sigma \log \sharp \sigma)$ time. Furthermore, we suppose that removing a vertex from $\sigma$ takes $O(\log \sharp \sigma)$ time.

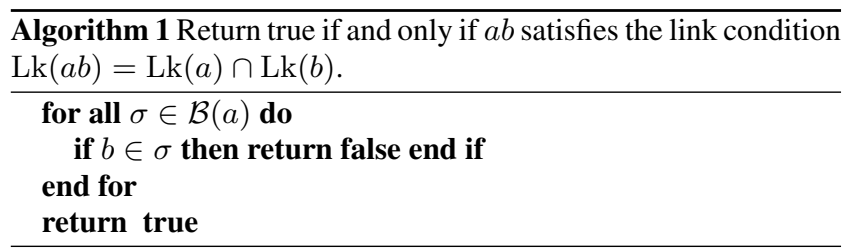

The time complexity for Algorithm 1 is $O\left(B_{a} \log d_{1}(a)\right)$ if the set of blockers $\mathcal{B}(a) \neq \emptyset$ and $O(1)$ otherwise.

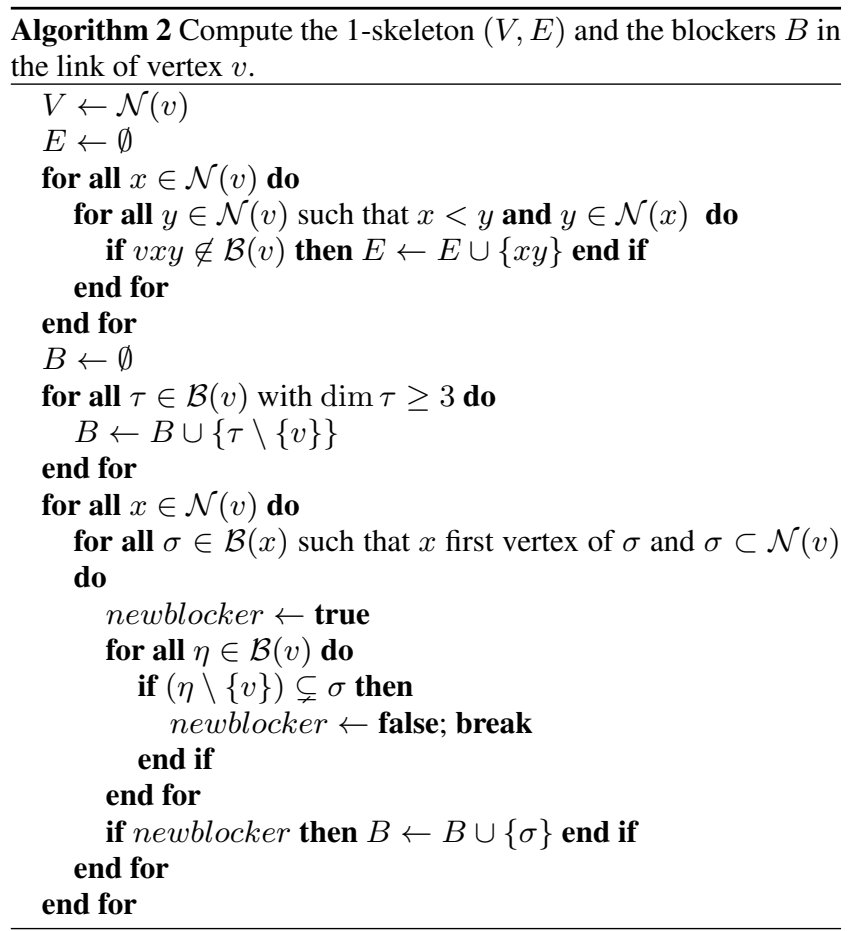

Algorithm 2 takes advantage of the fact that all simplices in the link of $v$ are subsets of $\mathcal{N}(v)$ and therefore blockers in the link of $v$ are to be found in $\mathcal{N}(v)$. The time complexity is $O(l(v))$ with

$$
\begin{aligned}
& l(v)=\left(N_{v}\right)^{2}\left(\log N_{\mathcal{N}(v)}+B_{v}\right)+ \\
& N_{v} B_{\mathcal{N}(v)}+M_{v} N_{v} \log N_{v}+M_{v}^{\prime} B_{v} N_{v} \log N_{v} .
\end{aligned}
$$

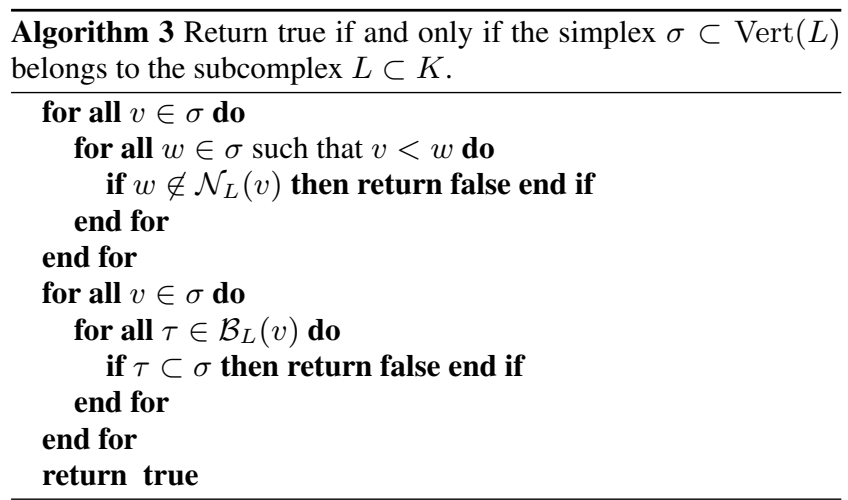

For $L=K$, the time complexity of Algorithm 3 is $O(g(\sharp \sigma, \sigma))$ where $g(x, y)=x^{2}\left(\log N_{y}+B_{y} \log x\right)$. If $L$ is the link of a vertex $v \in K$, the time complexity can also bound by $O(g(\sharp \sigma, \sigma))$ because for each vertex $x$ in the link of $v$, we have $\sharp \mathcal{N}_{\operatorname{Lk}(v)}(x) \leq$ $\sharp \mathcal{N}_{K}(x)$ and $\sharp \mathcal{B}_{\operatorname{Lk}(v)}(x) \leq \sharp \mathcal{B}_{K}(x)$.

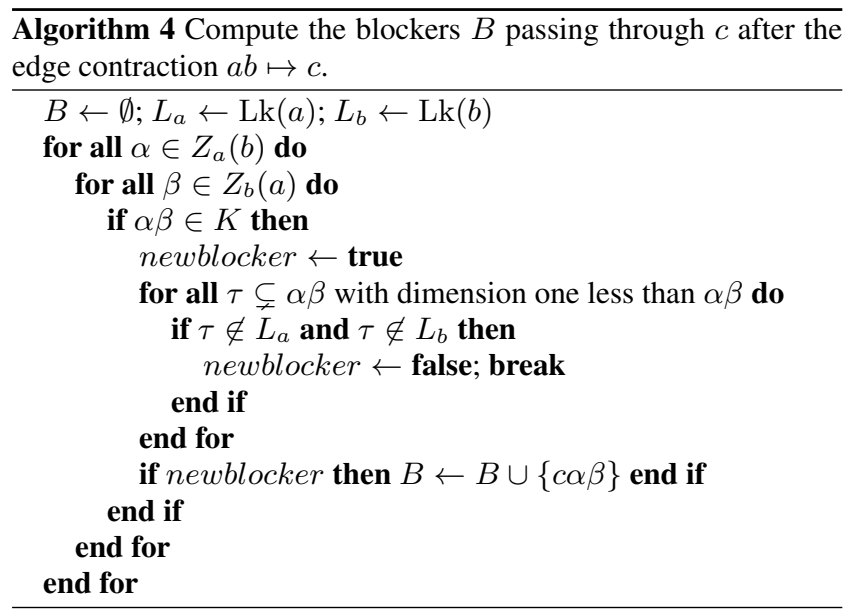

Let $d=d_{1}(c) \leq d_{0}(a)+d_{0}(b)$. Noting that the size of $Z_{a}(b)$ is upper bounded by $B_{b}+N_{a}$ and that $\alpha \beta \subset \operatorname{Lk}(a) \cup \operatorname{Lk}(b)$ has size $d$ at most, we get that the time complexity for Algorithm 4 is

$$
\begin{gathered}
O\left(l(a)+l(b)+\left(N_{a}+B_{b}\right)\left(N_{b}+B_{a}\right)\right. \\
{\left[g\left(d, \mathcal{N}_{a} \cup \mathcal{N}_{b}\right)+(d+1)\left(\log d+g\left(d-1, \mathcal{N}_{a}\right)+g\left(d-1, \mathcal{N}_{b}\right)\right]\right) .}
\end{gathered}
$$

We conclude the appendix by computing the complexities when there are no blockers through the vertices impacted by a local operation. The time complexity corresponding to Algorithm 1 , answering if an edge $a b$ in $K$ meets the link condition is $O(1)$. The time complexity corresponding to Algorithm 2 which builds a representation of the link of a vertex $v$, is $O\left(N_{v}^{2} \log N_{\mathcal{N}(v)}\right)$. For Algorithm 3 which tests whether a simplex $\sigma$ belongs to a subcomplex of $K$, we get $O\left((\sharp \sigma)^{2} \log N_{\sigma}\right)$ and updating the set of blockers takes $O\left(N_{a} N_{b} \log N_{\mathcal{N}(a)}\right)$ (or $O\left(N_{a} N_{b} \log N_{\mathcal{N}(b)}\right)$ ). These complexities give a good picture of the practical behavior of the simplification process when the number of blockers remains sufficiently small. 